\title{
Analysis of gas dynamics in a single-phase two-channel plasma torch at cold blowing and considering the interaction with the electric arc
}

\author{
Nikita Obraztsov ${ }^{1, *}$, Vladimir Frolov ${ }^{1}$, Mikhail Korotkikh ${ }^{1}$, and Ludmila Ushomirskaya ${ }^{1}$ \\ ${ }^{1}$ Peter the Great St. Petersburg Polytechnic University, 195251 St. Petersburg, Russia
}

\begin{abstract}
This article is devoted to the simulation of cold gas purge and flow taking into account the plasma arc in the channels of a single-phase two-channel high-voltage plasma torch with cylindrical electrodes. The results obtained in the process of modeling made it possible to simplify the model for further modeling, which will result in the distribution of velocities, temperatures and voltage drop on the arc depending on the time.
\end{abstract}

\section{Introduction}

The characteristics of low-temperature plasma generators depend on the interactions of plasma-forming working gas jets with electric arcs and on the organization of plasma-forming working gases. High-voltage AC plasma torches with power up to $50 \mathrm{~kW}$ and rod electrodes were developed and tested at the Institute for Institute for Electrophysics and Electric Power RAS (IEE RAS) [1-5]. The properties of electric arcs burning in cylindrical channels of plasma torches have not yet been fully studied, although a large amount of research work has been done to date [6-22]. A detailed study of these physical and chemical processes and their characteristics are relevant and timely for further efficiency improvement and practical application of the studied plasma torches, as their efficiency, reliability, stability, long-term operation and management of these processes are important engineering and practical tasks of subsonic gas flows. The solution of all these problems is impossible without the formulation of experimental studies aimed at a detailed study of physical and chemical processes and the identification of the role of gas-dynamic and kinetic aspects in order to build physical and mathematical models that adequately describe the observed and investigated phenomena. This requires a compelling justification and a reasonable application of experimental methods of structure study reacting flows, providing reliable information about the development of the process flow of the gas flow in the cylindrical channels of the investigated plasma torches with individual gaseous-vortex stabilization of the electric arc. At this stage of research, two models were performed in which the flow of a cold plasma-forming gas (argon) fed tangentially to the axial stabilization of the arc column in the plasma torch channel was taken into account and taking into account its interaction with the heated region of the cylindrical plasma torch channel.

\section{Model of plasma torch}

The object of study in this paper is a high-voltage AC plasma torch with gas-vortex stabilization of the arc (fig.1) [23]. Single-phase high-voltage AC plasma torch is a two-jet plasma torch with vortex stabilization of the arc. The body is made of stainless steel and is equipped with a water cooling jacket. It is a converging cylindrical channels. Each channel has a tangential gas supply to stabilize the arcs and protect the walls. Replaceable electrodes are made of a special alloy of iron and copper. When the operating voltage is applied to the electrodes in the areas of the minimum gap between them and the channel walls, an arc discharge is initiated. The formed electric arcs are blown by the flow of plasma-forming gas to the ends of the electrodes, stabilized and closed at the outlet of the channels.

\footnotetext{
* Corresponding author: nikita.obrazcov@mail.ru
} 


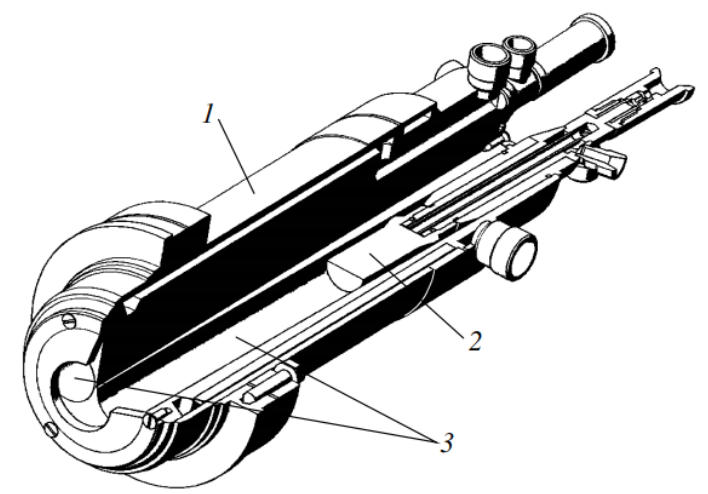

Fig. 1. High-voltage AC plasma torch with rod electrodes: 1-housing, 2-electrode, 3-channels

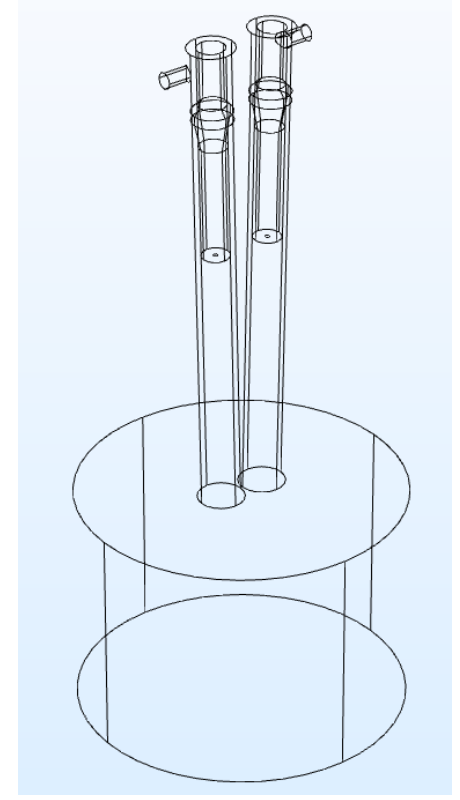

Fig. 2. The geometry of the plasma torch in Comsol Multuphysics

In Comsol, the geometry of the internal cavity of the plasma torch presented in Fig.2. The laminar flow module was used to model the flow. Basic equations of the stationary mathematical model in Comsol Multiphysics:

- Momentum equation:

$$
\rho(\boldsymbol{v} \nabla) \boldsymbol{v}=\boldsymbol{F}+\nabla\left[-p I+\mu\left(\nabla \boldsymbol{v}+(\nabla \boldsymbol{v})^{T}\right)\right]
$$

where $\boldsymbol{F}$ - forces acting on the plasma flow; $\boldsymbol{v}$ - plasma velocity vector; $I$-identity matrix; ; $\rho$ - temperature-dependent plasma density; $\mu$-temperature-dependent plasma viscosity.

- Continuity equation:

$$
\nabla(\rho \boldsymbol{v})=0
$$

Argon gas is supplied tangentially at a speed of $20 \mathrm{~m} / \mathrm{s}$ through the side inputs. Supply gas temperature $273 \mathrm{~K}$. At gas velocities below $20 \mathrm{~m} / \mathrm{s}$, it is obvious that the Mach number is less than 0.3 and the gas is incompressible. The Reynolds number does not exceed 2000 and the regime is laminar. Gravity and buoyancy force are not allowed for. The pressure and viscous dissipation force are neglected. On the walls of the plasma torch, non-flow conditions were set. At the boundaries of the lower cylinder, the atmospheric pressure condition is set. For the model with a burning arc, the temperature distribution shown in Fig.3. 


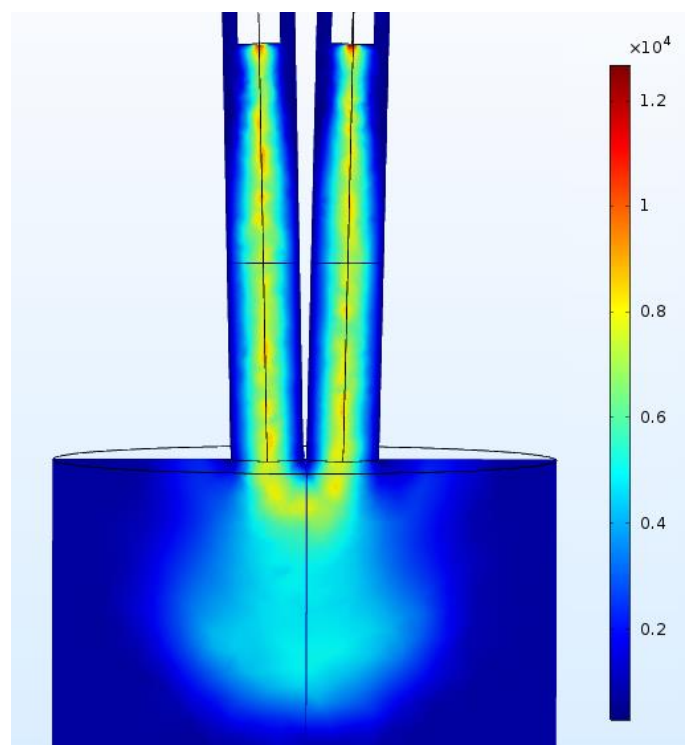

Fig. 3. The temperature distribution in the channels of the plasma torch

A stationary problem is considered, and we use Algebraic Multigrid Solver to solve it. To improve the convergence of the problem it is solved in several stages. The solution of the previous problem is used as initial conditions at the subsequent step. At the first stage we use an inconsistent stabilization with tuning parameter 0.5 and pseudo time stepping. Then the tuning parameter is reduced by 10 times. Then the stabilization is eliminated and at the last stage the pseudo time stepping is cancelled. The grid consists of 120,000 elements. The average calculation time of the model with all steps is about 3 hours.

\section{Results}

As a result of the modeling the velocity distributions in the channels of the plasma torch at argon flow at a temperature of $273 \mathrm{~K}$ and with regard to the temperature field (see Fig.3) were obtained. Fig.4 presents the current lines for two cases and it suggests the similar nature of the flows. An axis from the center of the electrode end face to the center of the channel outlet was chosen. The velocity distributions are shown in Fig.6 and Fig.7. From analysis of these relationships, it is clear that the flow patterns are similar. Return flows can be observed at the electrodes, in case of the burning arc the return flow is greater by $1 \mathrm{~m} / \mathrm{s}$ as compared to the cold purge, which is regular. The main contribution to the current is made by a z-axis velocity component.

Given that the plasma torch has a cooling jacket and the gas cools the walls and the electrode it was proposed to consider the XY cross section $10 \mathrm{~mm}$ above the electrode end. Fig.5 shows distributions of the velocity module in this cross section. At this distance the arc has no effect on the gas flow.

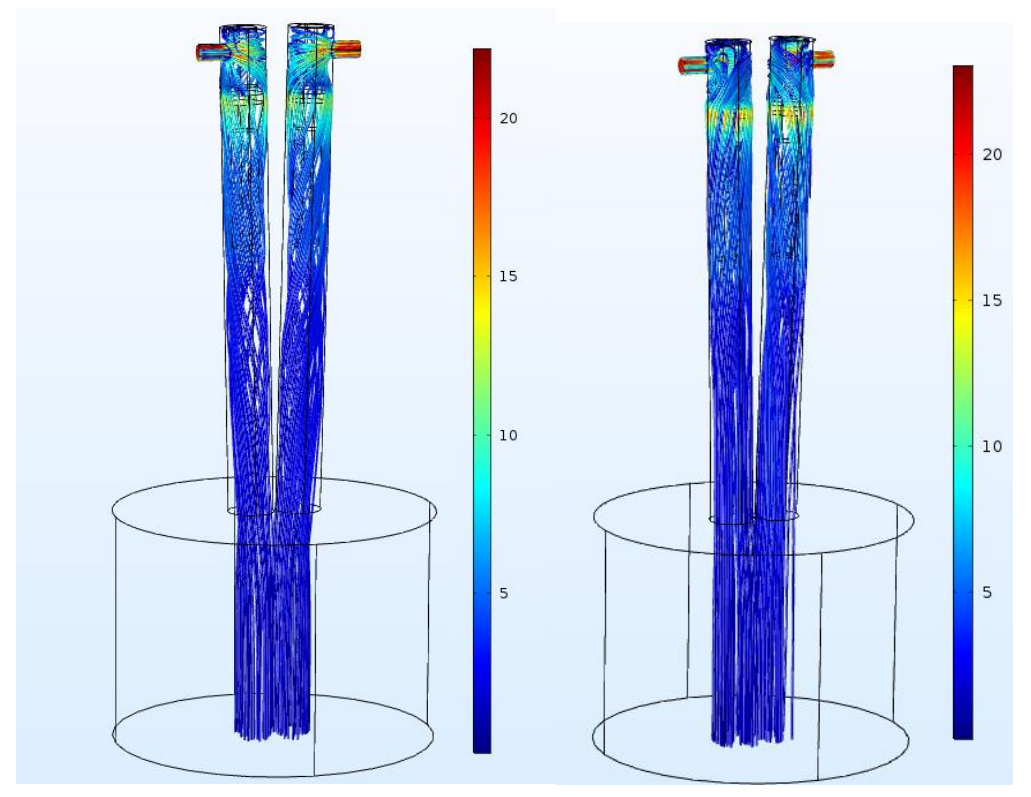

Fig. 4. Current lines (speed module). Cold purge on the left, with arc on the right 

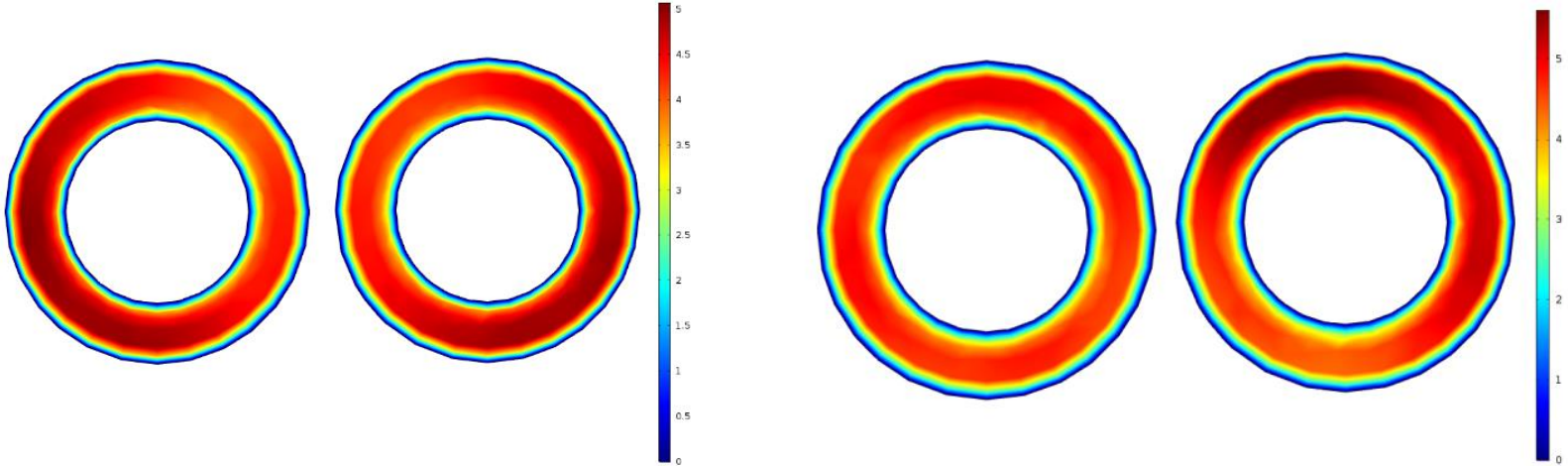

Fig. 5. Distribution of velocity module in $\mathrm{XY}$ cross section $10 \mathrm{~mm}$ above the end of the electrode. Cold purge on the left, with arc on the right

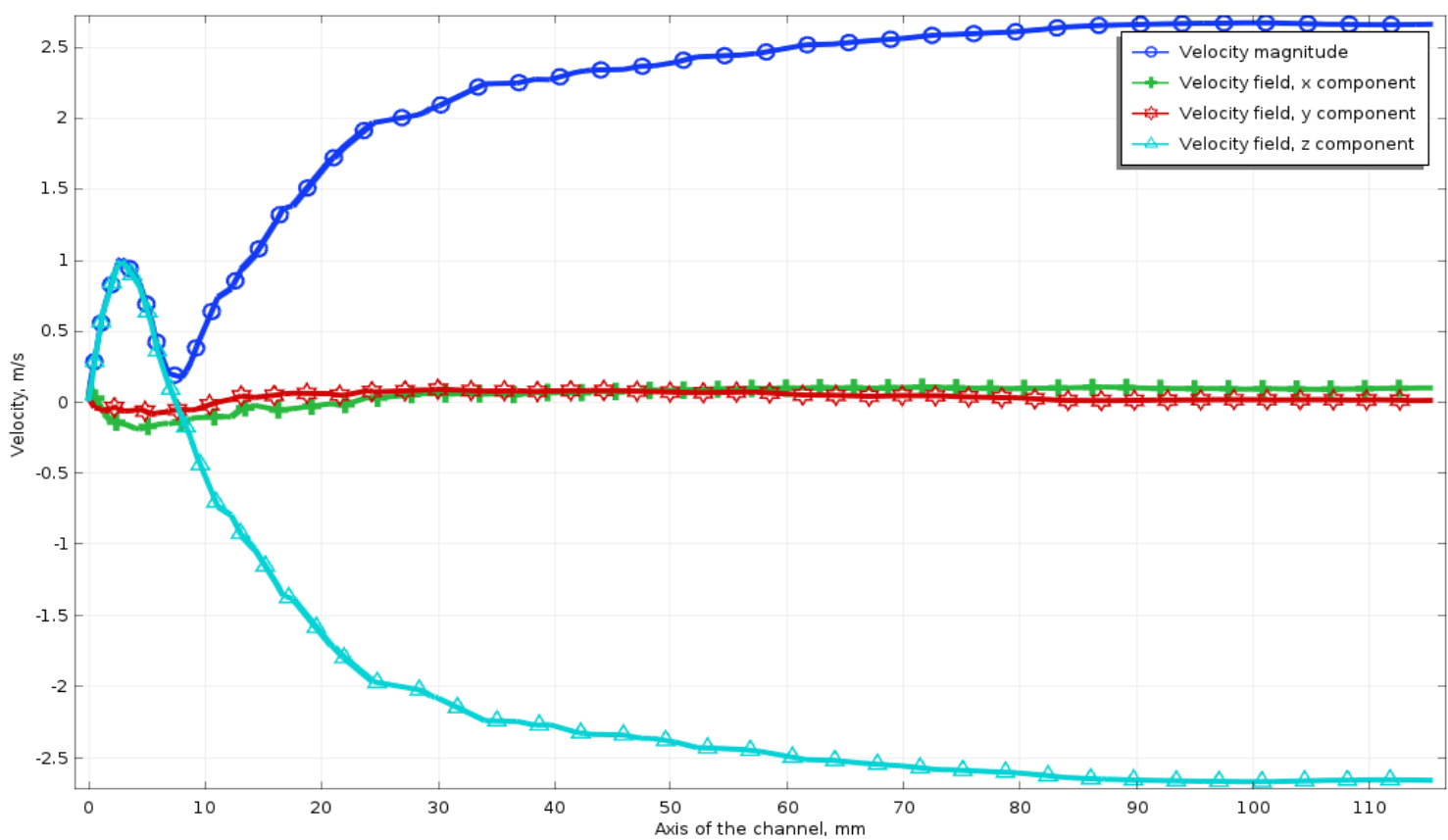

Fig. 6. Velocity distribution from the end of the electrode along the axis of the channel of the plasma torch. (Cold purge)

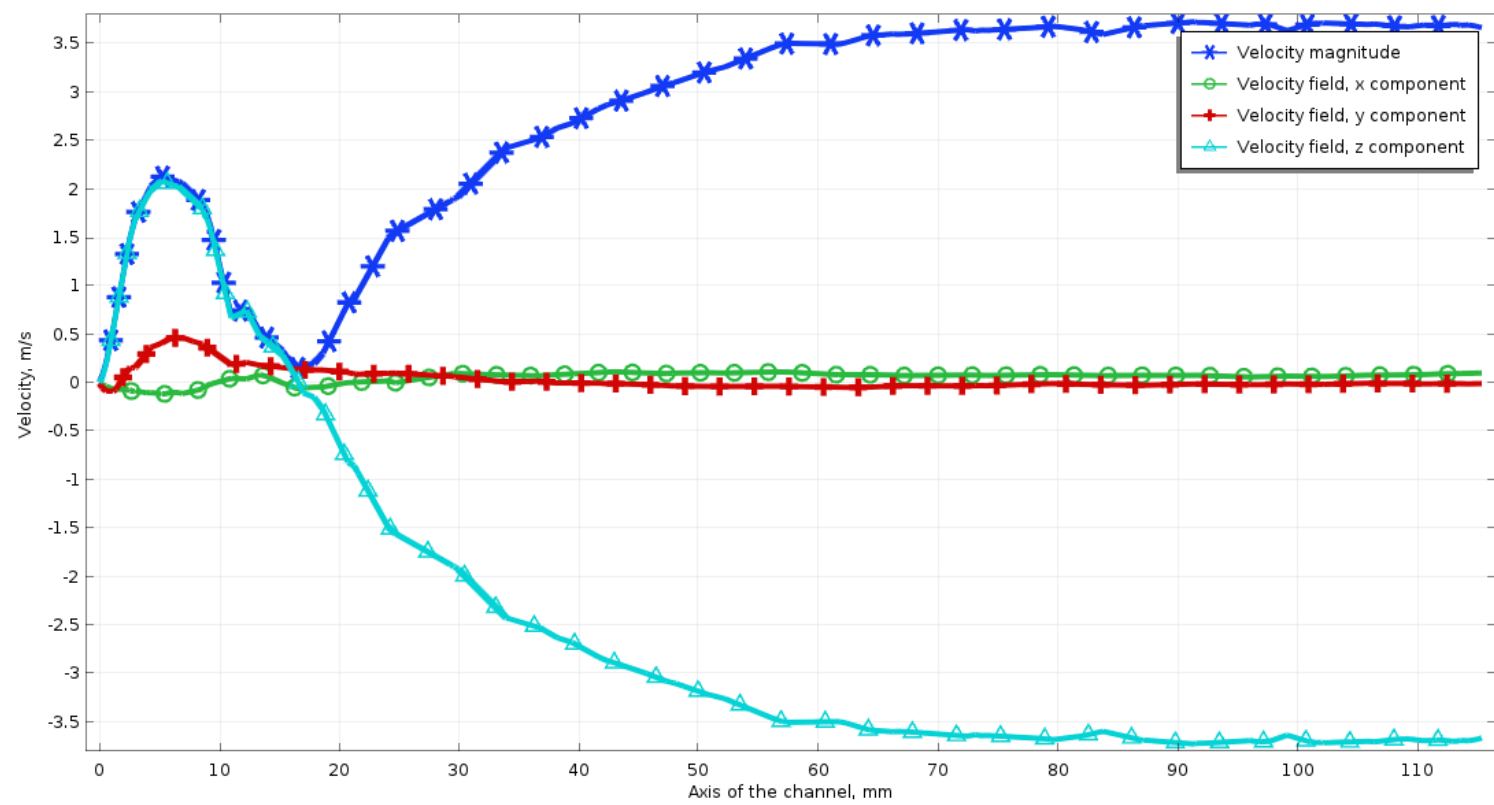

Fig. 7. Velocity distribution from the end of the electrode along the axis of the channel of the plasma torch. (Including arc) 


\section{Conclusions}

The purpose of this work was to simplify the simulation of plasma torches. Such tasks are especially difficult since for its solution it is necessary to take into account many interrelated physical processes. In addition, to solve such problems requires a large number of calculation resources. The literature presents many simplifications used for such tasks. This article proposes a different approach to simplify modeling. Simplification consists of preliminary consideration of the hydrodynamic problem and simplification of the geometry and obtaining initial data for the velocity field.

As a result of the modeling, velocity distributions were obtained for the case with gas (argon) with a temperature of 273 $\mathrm{K}$ in the channels of the plasma torch with a flow rate of $1 \mathrm{~g} / \mathrm{s}$ and with regard to the expected temperature field in the operating mode. When modeling the plasma devices, the most difficult task is to solve the hydrodynamics problem. The simulation showed that a return flow is observed $20 \mathrm{~mm}$ from the electrode end and in case of the burning arc, the rate of the return flow increases. The flow in the channel $10 \mathrm{~mm}$ electrode above the end face of the is also considered. This is necessary to obtain the velocity distributions for further simplification of the model. This will reduce the resources required for the model calculation. The next step will be creation of a magneto hydrodynamic model of an ac plasma generator.

\section{References}

1. F.G. Rutberg, A.A. Safronov, S. D. Popov, et al., High. Temp. 44 (205) (2006).

2. F.G. Rutberg., A.A. Safronov, G.V. Nakonechnyi, et al., Izv. Vyssh. Uchebn. Zaved. Fizika 9 (77) (2007)

3. A.V. Surov, S.D. Popov, E.O. Serba, et al. J. Phys. 927 (2017)

4. A.V. Surov, S.D. Popov, E.O. Serba, et al., J. Phys. 891 (2017)

5. A.V. Surov, S.D. Popov, E.O. Serba, et al., J. Phys. 825 (2017)

6. I. Murashov, The development of de plasma torch for the spraying technology taking into account the phenomena of plasma flow instability. (2016)

7. I.A. Glebov and F.G. Rutberg, Powerful plasma generators [in Russian].

8. A.M. Borovskoy, Usp.Prikl.Fiz., 450 (2013)

9. M.F. Zhukov, A.S. Archakov, V.P. Lukashov High-temperature flows and heat transfer: collection of articles 3 (46) (1990)

10. A.M. Borovskoy, S.D. Popov, A.V. Surov J. Phys. 461 (1) (2013)

11. N.V. Obraztsov, V.Ya. Frolov 2018 IEEE (eiconrus)

12. I. Murashov NTV 2 (243) (2016)

13. I. Murashov, V.Frolov, D. Ivanov 2016 IEEE (eiconrus)

14. A. Kadyrov, I. Murashov, V. Frolov Electricity (MEI) 5 (2018)

15. A.M. Borovskoy, Usp. Prikl. Fiz., 450 (2013).

16. A.M. Borovskoy, Prikl. Fiz., 21 (2014).

17. A.M. Borovskoy, Usp. Prikl. Fiz., 105 (2014).

18. A.M. Borovskoy, Prikl. Fiz., 40 (2014).

19. A.M. Borovskoy, Usp. Prikl. Fiz., 18 (2018).

20. C. Rehmet, V. Rohani, F. Cauneau, and L. Fulcheri, Plasma Chem. Plasma Process. 33 (2013).

21. C. Rehmet, F. Fabry, V. Rohani, F. Cauneau, and L. Fulcheri, Plasma Chem. Plasma Process. 34 (4) (2014).

22. L. Fulcheri, F. Fabry, S. Takali, and V. Rohani, Plasma Chem. Plasma Process. 35 (4) (2015).

23. F.G Rutberg, A.V. Pavlov, S.D. Popov, et al., High. Temp. 47 (2009). 